\title{
Constant-Weight Gray Codes for Local Rank Modulation
}

\author{
Eyal En Gad, Michael Langberg, Member, IEEE, Moshe Schwartz, Senior Member, IEEE and \\ Jehoshua Bruck, Fellow, IEEE
}

\begin{abstract}
We consider the local rank-modulation scheme in which a sliding window going over a sequence of real-valued variables induces a sequence of permutations. Local rankmodulation is a generalization of the rank-modulation scheme, which has been recently suggested as a way of storing information in flash memory.

We study constant-weight Gray codes for the local rankmodulation scheme in order to simulate conventional multi-level flash cells while retaining the benefits of rank modulation. We provide necessary conditions for the existence of cyclic and cyclic optimal Gray codes. We then specifically study codes of weight 2 and upper bound their efficiency, thus proving that there are no such asymptotically-optimal cyclic codes. In contrast, we study codes of weight 3 and efficiently construct codes which are asymptotically-optimal. We conclude with a construction of codes with asymptotically-optimal rate and weight asymptotically half the length, thus having an asymptotically-optimal charge difference between adjacent cells.
\end{abstract}

Index Terms-Gray code, rank modulation, local rank modulation, permutations, flash memory

\section{INTRODUCTION}

$\mathbf{I}$ $\mathrm{N}$ a recent series of papers [27], [28], [41], [44], the rankmodulation scheme was suggested as a way of storing information in flash-memory devices. Basically, instead of a conventional multi-level flash cell in which the charge level of a single cell is measured and quantized to a symbol from the input alphabet, in the rank-modulation scheme the permutation induced by the relative charge levels of several cells comprises the stored information. The scheme, first described in [27] in the context of flash memory, works in conjunction with a simple cell-programming operation called "push-to-the-top", which raises the charge level of a single cell above the rest of the cells. It was suggested in [27] that this scheme eliminates

The material in this paper was presented in part at the IEEE International Symposium on Information Theory (ISIT 2010), Austin, TX, U.S.A., June 2010, and at the 26-th IEEE Convention of Electrical ans Electronics Engineers in Israel (IEEEI 2010), Eilat, Israel, November 2010.

Eyal En Gad is with the Department of Electrical Engineering, California Institute of Technology, 1200 E. California Blvd., Mail Code 136-93, Pasadena, CA 91125, U.S.A. (e-mail: eengad@caltech.edu).

Michael Langberg is with the Computer Science Division, Open University of Israel, Raanana 43107, Israel (e-mail: mikel@openu.ac.il).

Moshe Schwartz is with the Department of Electrical and Computer Engineering, Ben-Gurion University, Beer Sheva 84105, Israel (e-mail: schwartz@ee.bgu.ac.il).

Jehoshua Bruck is with the Department of Electrical Engineering, California Institute of Technology, 1200 E. California Blvd., Mail Code 136-93, Pasadena, CA 91125, U.S.A. (e-mail: bruck@paradise.caltech.edu).

This work was supported in part by ISF grant 134/10, ISF grant 480/08, the Open University of Israel's research fund (grant no. 46114), the NSF grant ECCS-0802107, and an NSF-NRI award. the over-programming problem in flash memories, reduces corruption due to retention, and speeds up cell programming.

This is certainly not the first time permutations have been used for modulation purposes. Permutations have been used as codewords as early as the works of Slepian [39] (later extended in [2]), in which permutations were used to digitize vectors from a time-discrete memoryless Gaussian source, and Chadwick and Kurz [9], in which permutations were used in the context of signal detection over channels with non-Gaussian noise (especially impulse noise). Further early studies include works such as [2]-[4], [8], [12], [13]. More recently, permutations were used for communicating over powerlines (for example, see [43]), and for modulation schemes for flash memory [27], [28], [41], [44].

An important application for rank-modulation in the context of flash memory was described in [27]: A set of $n$ cells, over which the rank-modulation scheme is applied, is used to simulate a single conventional multi-level flash cell with $n$ ! levels corresponding to the alphabet $\{0,1, \ldots, n !-1\}$. The simulated cell supports an operation which raises its value by 1 modulo $n$ !. This is the only required operation in many rewriting schemes for flash memories (see [5], [24]-[26], [45]), and it is realized in [27] by a Gray code traversing the $n$ ! states where, physically, the transition between two adjacent states in the Gray code is achieved by using a single "pushto-the-top" operation.

Most generally, a gray code is a sequence of distinct elements from an ambient space such that adjacent elements in the sequence are "similar". Ever since their original publication by Gray [22], the use of Gray codes has reached a wide variety of areas, such as storage and retrieval applications [10], processor allocation [11], statistics [14], hashing [18], puzzles [20], ordering documents [30], signal encoding [31], data compression [34], circuit testing [35], and more. For a survey on Gray codes the reader is referred to [37].

A drawback to the rank-modulation scheme is the need for a large number of comparisons when reading the induced permutation from a set of $n$ cell-charge levels. Instead, in a recent work [44], the $n$ cells are locally viewed through a sliding window resulting in a sequence of small permutations which require less comparisons. We call this the local rankmodulation scheme. The aim of this work is to study Gray codes for the local rank-modulation scheme. The paper is organized as follows: In Section II the exact setting, notation, and definitions are presented. We study, in Section III, necessary conditions for the existence of Gray codes for our setting. In Section IV we give constructions for Gray codes of low 


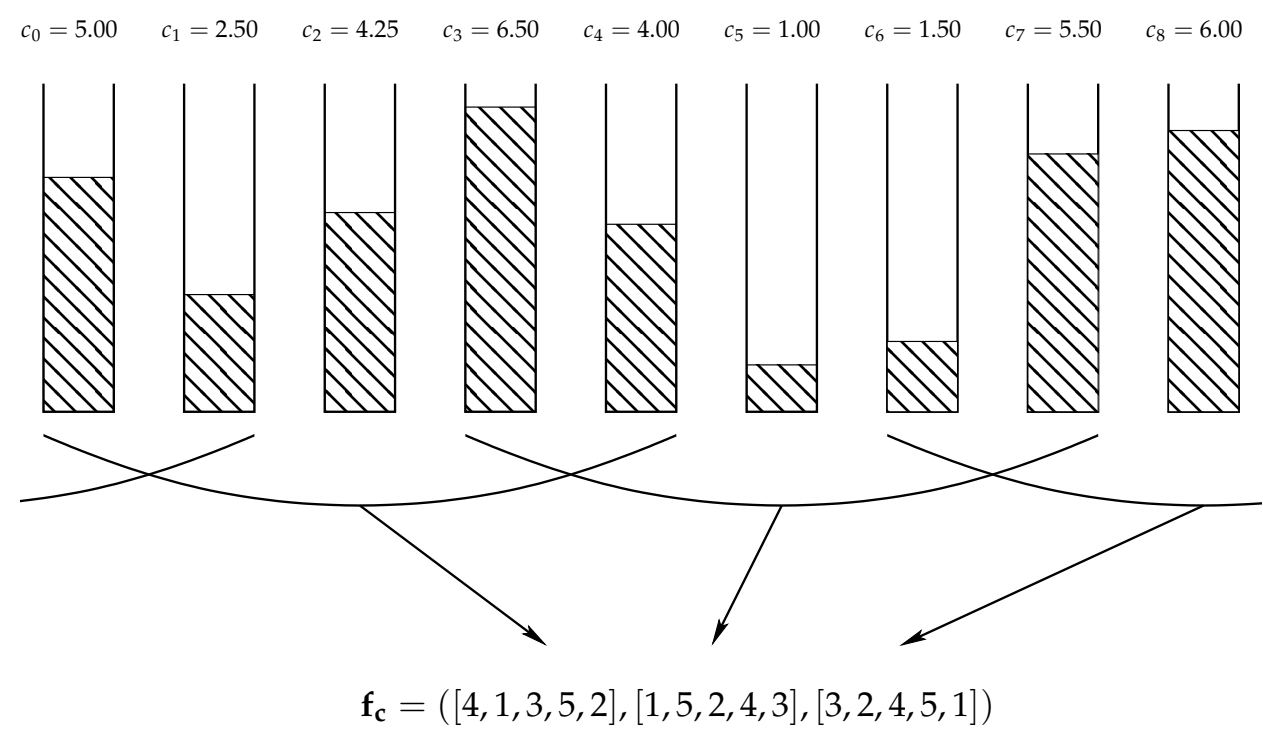

Figure 1. Demodulating a $(3,5,9)$-locally rank-modulated signal.

constant weight and study their efficiency. We turn, in Section $\mathrm{V}$, to construct Gray codes with asymptotically-optimal rates and weight asymptotically half the length. We conclude in Section VI with a summary and a set of open problems.

\section{DEFINITIONS AND NotATION}

\section{A. Local Rank Modulation}

Let us consider a sequence of $t$ real-valued variables, $\mathbf{c}=$ $\left(c_{0}, c_{1}, \ldots, c_{t-1}\right) \in \mathbb{R}^{t}$, where we further assume $c_{i} \neq c_{j}$ for all $i \neq j$. The $t$ variables induce a permutation $f_{\mathbf{c}} \in S_{t}$, where $S_{t}$ denotes the set of all permutations over $[t]=\{1,2, \ldots, t\}$. The permutation $f_{\mathfrak{c}}$ is uniquely defined by the constraints $c_{f_{\mathbf{c}}(i)-1}>c_{f_{\mathbf{c}}(j)-1}$ for all $i<j$, i.e., if we sort $\mathbf{c}$ in descending order, $c_{j_{1}}>c_{j_{2}}>\cdots>c_{j_{t}}$ then $f_{\mathbf{c}}(i)=j_{i}+1$ for all $1 \leqslant i \leqslant t$.

Given a sequence of $n$ variables, $\mathbf{c}=\left(c_{0}, c_{1}, \ldots, c_{n-1}\right)$, we define a window of size $t$ at position $p$ to be

$$
\mathbf{c}_{p, t}=\left(c_{p}, c_{p+1}, \ldots, c_{p+t-1}\right)
$$

where the indices are taken modulo $n$, and also $0 \leqslant p \leqslant n-1$, and $1 \leqslant t \leqslant n$.

We now define the $(s, t, n)$-local rank-modulation (LRM) scheme, which we do by defining the demodulation process. Let $s \leqslant t \leqslant n$ be positive integers, with $s \mid n$. Given a sequence of $n$ distinct real-valued variables, $\mathbf{c}=\left(c_{0}, c_{1}, \ldots, c_{n-1}\right)$, the demodulation maps $\mathbf{c}$ to the sequence of $n / s$ permutations from $S_{t}$ as follows:

$$
\mathbf{f}_{\mathbf{c}}=\left(f_{\mathbf{c}_{0, t}}, f_{\mathbf{c}_{s, t}}, f_{\mathbf{c}_{2 s, t}} \ldots, f_{\mathbf{c}_{n-s, t}}\right) .
$$

Loosely speaking, we scan the $n$ variables using windows of size $t$ positioned at multiples of $s$ and write down the permutations from $S_{t}$ induced by the local views of the sequence.

In the context of flash-memory storage devices, we shall consider the $n$ variables, $\mathbf{c}=\left(c_{0}, c_{1}, \ldots, c_{n-1}\right)$, to be the charge-level readings from $n$ flash cells. The demodulated sequence, $\mathbf{f}_{\mathfrak{c}}$, will stand for the original information which was stored in the $n$ cells. This approach will serve as the main motivation for this paper, as it was also for [27], [28], [41], [44]. See Figure 1 for an example.

Though of no consequence to the rest of the paper, we mention in passing, that the sequence of local permutations given in (1) may be quite a wasteful method of representation. A more compact form may be defined using the (mixedradix) factoradic notation (see [29] for the earliest-known definition, and [27] for a related use) in which the ith mostsignificant digit counts the number of elements to the right of the $i$ th-from-left element, which are of lower value. We then represent each of the local permutations $f_{\mathrm{c}_{i \cdot s, t}}$ using the $s$ most-significant digits in its factoradic notation. Thus, for example, the configuration of Figure 1 would be represented by $((3,0,1),(4,2,0),(0,2,2))$.

We say a sequence $\mathbf{f}$ of $n / s$ permutations over $S_{t}$ is $(s, t, n)$ $L R M$ realizable if there exists $\mathbf{c} \in \mathbb{R}^{n}$ such that $\mathbf{f}=\mathbf{f}_{\mathfrak{c}}$, i.e., it is the demodulated sequence of $\mathbf{c}$ under the $(s, t, n)$-LRM scheme. Except for the degenerate case of $s=t$, not every sequence is realizable.

When $s=t=n$, the $(n, n, n)$-LRM scheme degenerates into a single permutation from $S_{n}$. This was the case in most of the previous works using permutations for modulation purposes. A slightly more general case, $s=t<n$ was discussed by Ferriera et al. [19] in the context of permutation trellis codes, where a binary codeword was translated tuplewise into a sequence of permutation with no overlap between the tuples. Finally, the most general case was defined by Wang et al. [44] (though in a slightly different manner where indices are not taken modulo $n$, i.e., with no wrap-around). In [44], the sequence of permutations was studied under a charge-difference constraint called bounded rank-modulation, and mostly with parameters $s=t-1$, i.e., an overlap of one position between adjacent windows.

Finding out the induced permutation from a sequence of $t$ real-valued readings requires at least $\Omega(t \log t)$ comparisons. 
Thus, to get the simplest hardware implementation we will consider the case of $t=2$ throughout the paper. The only non-trivial case to consider is therefore $s=1$, i.e., a $(1,2, n)$ LRM scheme. Demodulated sequences of permutations in this scheme contain only the permutations $[1,2]$ and $[2,1]$, and a single comparison between the charge levels of two adjacent flash memory cells is required to find the permutation. We will conveniently associate the logical value 1 with the permutation $[1,2]$, and 0 with $[2,1]$, thus forming a simple mapping between length $n$ binary sequences and permutation sequences from the $(1,2, n)$-LRM scheme. It is easily seen that the only two binary sequences not mapped to $(1,2, n)$ LRM sequences are the all-ones and all-zeros sequences.

\section{B. Gray Codes for $(1,2, n)-L R M$}

Generally speaking, a Gray code, G, is a sequence of distinct states (codewords), $G=g_{0}, g_{1}, \ldots, g_{N-1}$, from an ambient state space, $g_{i} \in S$, such that adjacent states in the sequence differ by a "small" change. What constitutes a "small" change usually depends on the code's application.

Since we are interested in building Gray codes for flash memory devices with the $(1,2, n)$-LRM scheme, our ambient space, which we denote as $S(n)$, is the set of all realizable sequences under $(1,2, n)$-LRM. This is simply the set of all the binary sequences of length $n$, excluding the all-ones and all-zeros sequences, i.e.,

$$
S=S(n)=\{0,1\}^{n}-\left\{0^{n}, 1^{n}\right\} .
$$

Each of the codewords, $g_{i} \in G$, is a string of $n$ bits which we shall denote as $g_{i}=g_{i, 0}, g_{i, 1}, \ldots, g_{i, n-1}$. Throughout the paper we will assume the index $j$ in $g_{i, j}$ is taken modulo $n$, and when appropriate, the index $i$ is taken modulo $N$.

The transition between adjacent states in the Gray code is directly motivated by the flash memory application, and was previously described and used in [27]. This transition is the "push-to-the-top" operation, which takes a single flash cell and raises its charge level above all others.

In our case, however, since we are considering a local rankmodulation scheme, the "push-to-the-top" operation merely raises the charge level of the selected cell above those cells which are comparable with it. As the window size is $t=2$, these cells are the ones directly before and after the selected cell. Thus, we define the set of allowed transitions as $T=\left\{\tau_{0}, \tau_{1}, \ldots, \tau_{n-1}\right\}$, which is a set of functions, $\tau_{j}: S \rightarrow S$, where $\tau_{j}$ represents a "push-to-the-top" operation performed on the $j$-th cell. If $v=v_{0} v_{1} \ldots v_{n-1} \in S(n)$, then $v^{\prime}=v_{0}^{\prime} v_{1}^{\prime} \ldots v_{n-1}^{\prime}=\tau_{j}(v)$ if

$$
v_{k}^{\prime}= \begin{cases}0 & k=j \\ 1 & k \equiv j+1 \quad(\bmod n) \\ v_{k} & \text { otherwise. }\end{cases}
$$

Loosely speaking, a transition is made by selecting a window of size 2 in the original codeword, and overwriting it with 01. See Figure 2 for an example.

Definition 1. A Gray code, $G$, for $(1,2, n)$-LRM (denoted $(1,2, n)$-LRMGC) is a sequence of distinct length- $n$ binary

$$
c_{0}=5.00 \quad c_{1}=2.50 \quad c_{2}=4.25 \quad c_{3}=6.50 \quad c_{4}=4.00 \quad c_{5}=1.00
$$

(a)

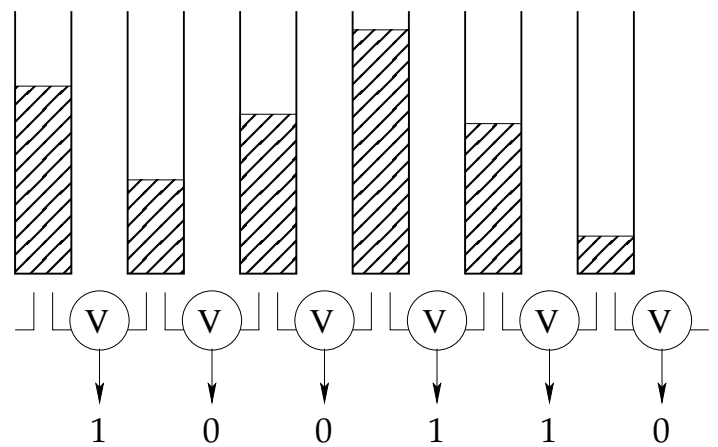

$$
c_{0}=5.00 \quad c_{1}=5.50 \quad c_{2}=4.25 \quad c_{3}=6.50 \quad c_{4}=4.00 \quad c_{5}=1.00
$$

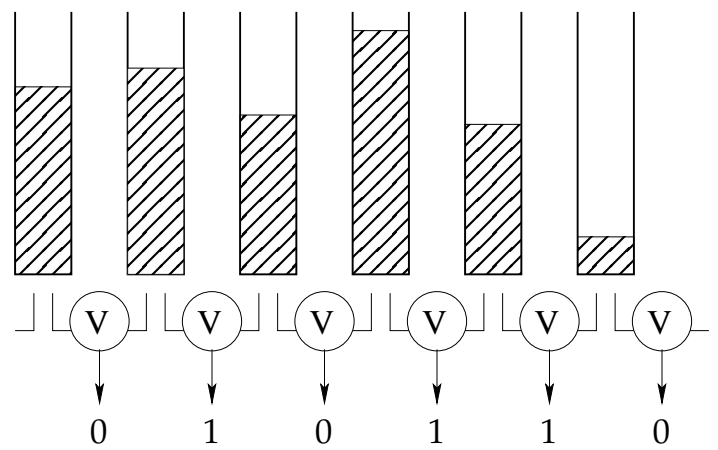

Figure 2. An example of a local "push-to-the-top" operation in a $(1,2,6)$ LRM scheme. The snapshot (a) presents the system before the change, while (b) presents the system after the change, which locally pushed $c_{1}$ above $c_{0}$ and $c_{2}$, thus changing the first two bits of the demodulated sequence.

codewords, $G=g_{0}, g_{1}, \ldots, g_{N-1}$, where $g_{i} \in S(n)$. For all $0 \leqslant i \leqslant N-2$, we further require that $g_{i+1}=\tau_{j}\left(g_{i}\right)$ for some $j$. If $g_{0}=\tau_{j}\left(g_{N-1}\right)$ for some $j$, then we say the code is cyclic. We call $N$ the size of the code, and say $G$ is optimal if $N=2^{n}-2=|S(n)|$.

When we perform a "push-to-the-top" operation on the $j$-th cell, let us denote its initial charge level as $c_{j}$, and its resulting charge level as $c_{j}^{\prime}$. We set $c_{j}^{\prime}$ to $\max \left\{c_{j-1}, c_{j+1}\right\}+\delta$, where $\delta>0$. Two important issues of concern are the difference in charge levels involved in a "push-to-the-top" operation, and cell saturation. In the former, the higher $c_{j}^{\prime}-c_{j}$ is, the more risk of disturbing neighboring cells, while in the latter, the higher we set $c_{j}^{\prime}$, the less number of updates to the cell before it saturates. Both concerns benefit from a value of $\delta$ as low as possible. Let us assume that a limited resolution exists and thus $\delta$ is bounded from below by a constant, which w.l.o.g., we can assume is 1 (after a proper scaling).

Let us now assume an optimal setting in which a "push-to-the-top" operation on the $j$-th cell sets $c_{j}^{\prime}=$ $\max \left\{c_{j-1}, c_{j+1}\right\}+1$. A general $(1,2, n)$-LRMGC may result in $c_{j}^{\prime}-c_{j}$ exponential in $n$, for some transition from $g_{i}$ to $g_{i+1}$. The same motivation in the case of $(n, n, n)$-LRM was discussed in [27], where a balanced variant of Gray codes was constructed to avoid the problem. We present a different variant of Gray codes to address the same issue.

First, for any binary string $v=v_{0} v_{1} \ldots v_{n-1}$, we call the 
number of 1's in $v$ the weight of $v$ and denote it as $\mathrm{wt}(v)$. We also denote by $S(n, w)$ the set of length- $n$ binary strings of weight $w$. We now define our variant of Gray codes:

Definition 2. A constant-weight Gray code for $(1,2, n)$-LRM (denoted $(1,2, n ; w)$-LRMGC), $G=g_{0}, g_{1}, \ldots, g_{N-1}$, is a Gray code for $(1,2, n)-L R M$ for which $g_{i} \in S(n, w)$ for all $0 \leqslant i \leqslant N-1$.

Definition 3. Let $G$ be a $(1,2, n ; w)-L R M G C$ of size $N$. We define the rate of the code as $R(G)=\frac{\log _{2} N}{n}$. The efficiency of $G$ is defined as $\operatorname{Eff}(G)=N /\left(\begin{array}{c}n \\ w\end{array}\right)$. If $\operatorname{Eff}(G)=1$ then we say $G$ is optimal. If $\operatorname{Eff}(G)=1-o(1)$, where $o(1)$ denotes a function that tends to 0 as $n \rightarrow \infty$, then we say $G$ is asymptotically optimal.

The transitions between adjacent states in the constantweight variant take on a very simple form: a window of size 2 in $g_{i}$ which contains 10 is transformed in $g_{i+1}$ into 01, i.e., "pushing" a logical 1 a single place to the right. Since we are interested in creating cyclic counters, we will be interested in cyclic Gray codes. An example of a cyclic optimal Gray code is given in Table I.

TABLE I

A CYCLIC OPTIMAL $(1,2,5 ; 2)$-LRMGC (THE CHANGED POSITIONS ARE UNDERLINED.)

$\begin{array}{lllll}\frac{1}{1} & 1 & 0 & 0 & \underline{0} \\ \underline{0} & \frac{0}{1} & \frac{1}{1} & 0 & 0 \\ 0 & \frac{1}{1} & \underline{0} & \underline{1} & 0 \\ 0 & \underline{0} & \underline{1} & \frac{1}{0} & 0 \\ 0 & 0 & 1 & \underline{0} & \underline{1} \\ 0 & 0 & \underline{0} & \underline{1} & 1 \\ \underline{1} & 0 & 0 & 1 & \underline{0} \\ \underline{1} & 0 & 0 & \underline{0} & \underline{1} \\ \underline{0} & \underline{1} & 0 & 0 & 1\end{array}$

It should be noted that Gray codes with a weaker restriction, allowing a 01 to be changed into 10 and also 10 to be changed back into 01, i.e., a 1 may be pushed either to the right or to the left, have been studied in the past [6], [7], [16], [23], [36].

We can show that under the constant-weight restriction, for any "push-to-the-top" operation,

$$
c_{j}^{\prime}-c_{j} \leqslant\left\lceil\frac{\max \{w, n-w\}}{\min \{w, n-w\}}\right\rceil .
$$

This is done by first assuming $2 w \leqslant n$, or else we flip all the bits and reverse the codewords. We will only use integer charge levels, and thus for any codeword, $g_{i}, \operatorname{wt}\left(g_{i}\right)=w$, we can find a realization by setting $c_{j+1}-c_{j}=1$ if $g_{i, j}=0$, and $c_{j+1}-c_{j}=-[(n-w) / w]$ if $g_{i, j}=1$, where $[\cdot]$ denotes either $\lfloor\cdot\rfloor$ or $\lceil\cdot\rceil$.

It is now easily shown by induction that a "push-to-the-top" operation on the $j$-th cell preserves charge-level differences between adjacent cells and only rearranges their order: by the induction hypothesis, initially we have $c_{j}-c_{j-1}=-[(n-$ $w) / w]$ and $c_{j+1}-c_{j}=1$. The "push-to-the-top" operation sets $c_{j}^{\prime}=\max \left\{c_{j-1}, c_{j+1}\right\}+1=c_{j-1}+1$ and then $c_{j}^{\prime}-$ $c_{j-1}=1$ and $c_{j+1}-c_{j}^{\prime}=-[(n-w) / w]$.

\section{NeCESSARY CONDITIONS}

We first present a simple necessary condition for the existence of a cyclic Gray code, and then expand it in the case of cyclic optimal codes. We use a coloring argument in the following way: We color the words in $S(n, w)$ using $n$ colors. We then show that in a cyclic Gray code all colors appear, and do so in equal amounts. We then follow with an analysis of the distribution of colors in $S(n, w)$, showing that in many cases they are not equally distributed, and hence no cyclic optimal code exists.

Definition 4. For any $v=v_{0} v_{1} \ldots v_{n-1} \in S(n, w)$, we define the first moment of $v$ as

$$
\chi(v)=\sum_{j=0}^{n-1} j \cdot v_{j}
$$

and the color of $v$ as $\chi(v) \bmod n$.

Theorem 5. Let $G$ be a cyclic $(1,2, n ; w)-L R M G C$ of size $N$. Then $n \mid N$.

Proof: If $v, v^{\prime} \in S(n, w)$ and $v^{\prime}=\tau_{j}(v)$ for some $j$, then it easily follows that $\chi\left(v^{\prime}\right) \equiv \chi(v)+1(\bmod n)$. Let us now denote $G=g_{0}, g_{1}, \ldots, g_{N-1}$. By the previous argument, $i \equiv i^{\prime}(\bmod n)$ iff $\chi\left(g_{i}\right) \equiv \chi\left(g_{i^{\prime}}\right)(\bmod n)$. Since the code is cyclic, necessarily $N \equiv 0(\bmod n)$.

We can use Theorem 5 to rule out the existence of cyclic optimal codes in certain cases.

Theorem 6. If $w$ is a prime, then there are no cyclic optimal $(1,2, n ; w)-L R M G C$ for which $\operatorname{gcd}(n, w) \neq 1$.

Proof: By the assumptions, necessarily $\operatorname{gcd}(n, w)=w$, and so $w \mid n$. For any $a, p \in \mathbb{N}, p$ prime, let us denote by $a_{p}$ the exponent of $p$ in the factorization of $a$. We can see that $N=\left(\begin{array}{c}n \\ w\end{array}\right)=\frac{n(n-1) \ldots(n-w+1)}{w !}$ and therefore $N_{w}=n_{w}-1$. But then $n \nmid N$ as required by Theorem 5 .

The divisibility condition set in Theorem 5 is not strong enough. For example, if we take $n=12$ and $w=6$, then indeed $12 \mid\left(\begin{array}{c}12 \\ 6\end{array}\right)$, and the possible existence of a cyclic optimal code with these parameters is not ruled out. However, by the conditions described in Corollary 7 and Lemma 8 it is ruled out.

Corollary 7. If a cyclic optimal $(1,2, n ; w)-L R M G C$ exists, then there are exactly $\left(\begin{array}{c}n \\ w\end{array}\right) / n$ strings of each color in $S(n, w)$.

Proof: By Theorem 5 we have $n \mid\left(\begin{array}{c}n \\ w\end{array}\right)$. Furthermore, by the proof of that theorem the code contains an equal number of codewords of each color. Since the code is optimal, i.e., covers all the strings of $S(n, w)$, the claim follows.

To be able to use the last corollary we count the exact number of strings of each color in $S(n, w)$. Though a solution may be deduced from a related theorem due to von Sterneck (see [15], Ch. II), we describe a cleaner self-contained solution, which is an extension of Sloane's method in [40]. In the following, let

$$
A_{n}(j, k)=|\{v \in S(n, w) \mid \chi(v) \equiv j, k \equiv n-w(\bmod n)\}|
$$

for all $0 \leqslant j, k \leqslant n-1$. Also, let $\phi$ stand for Euler's totient function, and $\mu$ stand for the Möbius function. 
Lemma 8. The number of strings from $S(n, w), 1 \leqslant w \leqslant n-$ 1 , of color $0 \leqslant a \leqslant n-1$, is given by

$$
A_{n}(a, n-w)=\frac{1}{n} \sum_{\substack{d|n \\
d| w}}(-1)^{\frac{w(d+1)}{d}} \phi(d) \frac{\mu\left(\frac{d}{\operatorname{gcd}(d, a)}\right)}{\phi\left(\frac{d}{\operatorname{gcd}(d, a)}\right)}\left(\begin{array}{c}
n / d \\
w / d
\end{array}\right) .
$$

Proof: We define the following generating function:

$$
f(x, y)=\sum_{j=0}^{n-1} \sum_{k=0}^{n-1} A_{n}(j, k) x^{j} y^{k} .
$$

An important observation that follows from the definition of $A_{n}(j, k)$ is that

$$
f(x, y)=\prod_{m=0}^{n-1}\left(x^{m}+y\right) \quad \bmod \left\langle x^{n}-1, y^{n}-1\right\rangle .
$$

Let $\xi=e^{\frac{2 \pi i}{n}} \in \mathbb{C}$ be an $n$-th complex root of unity, then

$$
f\left(\xi^{j}, \xi^{k}\right)=\sum_{j^{\prime}=0}^{n-1} \sum_{k^{\prime}=0}^{n-1} A_{n}\left(j^{\prime}, k^{\prime}\right) \xi^{j^{\prime} j} \xi^{k^{\prime} k} .
$$

Using the inverse two-dimensional discrete Fourier transform we get,

$$
A_{n}(j, k)=\frac{1}{n^{2}} \sum_{j^{\prime}=0}^{n-1} \sum_{k^{\prime}=0}^{n-1} f\left(\xi^{j^{\prime}}, \xi^{k^{\prime}}\right) \xi^{-j^{\prime} j} \xi^{-k^{\prime} k} .
$$

Let us denote $g=\operatorname{gcd}\left(n, j^{\prime}\right)$. We can directly calculate

$$
\begin{aligned}
f\left(\xi^{j^{\prime}}, \xi^{k^{\prime}}\right) & =\prod_{m=0}^{n-1}\left(\xi^{k^{\prime}}+\xi^{m j^{\prime}}\right) \\
& =(-1)^{n} \prod_{m=0}^{\frac{n}{g}-1}\left(-\xi^{k^{\prime}}-\xi^{m j^{\prime}}\right)^{g} \\
& =(-1)^{n}\left(\left(-\xi^{k^{\prime}}\right)^{\frac{n}{g}}-1\right)^{g} \\
& =\sum_{m=0}^{g}\left(\begin{array}{c}
g \\
m
\end{array}\right)(-1)^{(g-m)\left(\frac{n}{g}+1\right)} \xi^{k^{\prime} m \frac{n}{g}},
\end{aligned}
$$

where the third equality follows from the well-known fact that $\prod_{i=0}^{n}\left(z-\xi^{i}\right)=z^{n}-1$. It now follows that

$$
\begin{aligned}
\sum_{k^{\prime}=0}^{n-1} f\left(\xi^{j^{\prime}}, \xi^{k^{\prime}}\right) \xi^{-k^{\prime} k} & = \\
= & \sum_{m=0}^{g}\left(\begin{array}{c}
g \\
m
\end{array}\right)(-1)^{(g-m)\left(\frac{n}{g}+1\right)} \sum_{k^{\prime}=0}^{n-1} \xi^{k^{\prime}\left(m \frac{n}{g}-k\right)} .
\end{aligned}
$$

Since we are interested only in $1 \leqslant k \leqslant n-1$, it follows that $-(n-1) \leqslant k-m \frac{n}{g} \leqslant n-1$, and therefore

$$
\sum_{k^{\prime}=0}^{n-1} \xi^{k^{\prime}\left(m \frac{n}{g}-k\right)}= \begin{cases}0 & k \neq m \frac{n}{g} \\ n & k=m \frac{n}{g} .\end{cases}
$$

Substituting back into (4), we get for all $1 \leqslant k \leqslant n-1$

$$
\sum_{k^{\prime}=0}^{n-1} f\left(\xi^{j^{\prime}}, \xi^{k^{\prime}}\right) \xi^{-k^{\prime} k}= \begin{cases}0 & \frac{n}{g} \nmid k \\ \left.(-1)^{\left(g-k \frac{g}{n}\right)\left(\frac{n}{g}+1\right)} \underset{k g / n}{g}\right) n & \frac{n}{g} \mid k .\end{cases}
$$

We again substitute the result back into (3) and summing by divisors of both $n$ and $k$, we get

$$
A_{n}(j, k)=\frac{1}{n^{2}} \sum_{\substack{d|n \\
d| k}}(-1)^{\frac{(n-k)(d+1)}{d}}\left(\begin{array}{l}
n / d \\
k / d
\end{array}\right) n \sum_{\substack{m=1 \\
\operatorname{gcd}(m, d)=1}}^{d} \xi^{-j m} .
$$

The inner sum is a Ramanujan sum (see [1]) which equals

$$
\sum_{\substack{m=1 \\ \operatorname{gcd}(m, d)=1}}^{d} \xi^{-j m}=\phi(d) \frac{\mu\left(\frac{d}{\operatorname{gcd}(d, j)}\right)}{\phi\left(\frac{d}{\operatorname{gcd}(d, j)}\right)}
$$

thus getting

$$
A_{n}(j, k)=\frac{1}{n} \sum_{\substack{d|n \\
d| k}}(-1)^{\frac{(n-k)(d+1)}{d}} \phi(d) \frac{\mu\left(\frac{d}{\operatorname{gcd}(d, j)}\right)}{\phi\left(\frac{d}{\operatorname{gcd}(d, j)}\right)}\left(\begin{array}{l}
n / d \\
k / d
\end{array}\right) .
$$

A simple rewriting of the last expression gives the desired result.

Returning to the previous example of $n=12$ and $w=6$ we can now use Lemma 8 to find that there are $A_{12}(0,12-6)=$ 76 words in $S(12,6)$ colored 0 , while there are $A_{12}(1,12-$ $6)=78$ words colored 1 . Thus, by Corollary 7 no optimal cyclic code is possible. The following theorem may be thought of as an extension of Theorem 6 to the case of $w$ not a prime.

Theorem 9. For any $w \in \mathbb{N}$ there exists $n_{0}(w) \in \mathbb{N}$ such that for all $n>n_{0}(w)$, there is no cyclic optimal $(1,2, n ; w)$ LRMGCs unless $\operatorname{gcd}(n, w)=1$.

Proof: Fix a weight $w$. We will show that there exists $n_{0}(w)$ such that for all $n>n_{0}(w), \operatorname{gcd}(n, w) \neq 1$, there is no cyclic optimal $(1,2, n ; w)$-LRMGC. We will do so by showing that $A_{n}(0, n-w) \neq A_{n}(1, n-w)$.

Let $p \geqslant 2$ denote the smallest prime number such that $p \mid \operatorname{gcd}(n, w) \neq 1$. We shall also need the fact that

$$
\left(\begin{array}{l}
a \\
b
\end{array}\right)=\frac{a}{b}\left(\begin{array}{l}
a-1 \\
b-1
\end{array}\right) .
$$

Now,

$$
\begin{aligned}
& A_{n}(0, n-w)-A_{n}(1, n-w)= \\
& =\frac{1}{n} \sum_{\substack{d|n \\
d| w}}(-1)^{\frac{w(d+1)}{d}}(\phi(d)-\mu(d))\left(\begin{array}{c}
n / d \\
w / d
\end{array}\right) \\
& =(-1)^{w(p+1) / p} \cdot \frac{p}{n}\left(\begin{array}{c}
n / p \\
w / p
\end{array}\right)+ \\
& \quad \frac{1}{n} \sum_{d \mid \operatorname{gcd}(n, w)}(-1)^{\frac{w(d+1)}{d}}(\phi(d)-\mu(d))\left(\begin{array}{c}
n / d \\
w / d
\end{array}\right) .
\end{aligned}
$$

We shall proceed to show that, for large enough $n$,

$$
p\left(\begin{array}{c}
n / p \\
w / p
\end{array}\right)>\left|\sum_{\substack{\operatorname{gcd}(n, w) \\
d>p}}(-1)^{\frac{w(d+1)}{d}}(\phi(d)-\mu(d))\left(\begin{array}{c}
n / d \\
w / d
\end{array}\right)\right|
$$


which will prove our claim. Indeed, set $n_{0}=\frac{w^{3}}{2}$, and then for all $n>n_{0}$

$$
\begin{aligned}
\mid \sum_{\substack{\operatorname{gcd}(n, w) \\
d>p}}(-1)^{\frac{w(d+1)}{d}} & (\phi(d)-\mu(d))\left(\begin{array}{c}
n / d \\
w / d
\end{array}\right) \mid \leqslant \\
& \leqslant \sum_{\substack{d \mid \operatorname{gcd}(n, w) \\
d>p}}(\phi(d)-\mu(d))\left(\begin{array}{c}
n / d \\
w / d
\end{array}\right) \\
& \leqslant \sum_{d=1}^{w} w\left(\begin{array}{c}
n / p-1 \\
w / p-1
\end{array}\right)=\frac{w^{3}}{n}\left(\begin{array}{c}
n / p \\
w / p
\end{array}\right) \\
& <2\left(\begin{array}{c}
n / p \\
w / p
\end{array}\right) \leqslant p\left(\begin{array}{c}
n / p \\
w / p
\end{array}\right),
\end{aligned}
$$

as we claimed.

It should be noted that a more careful analysis can reduce the value of $n_{0}$ in the proof of Theorem 9 . We also observe that when $\operatorname{gcd}(n, w)=1$, all strings of length $n$ and weight $w$ have full cyclic period. If $v, v^{\prime} \in S(n, w)$ and $v^{\prime}$ is a cyclic shift to the right of $v$, then $\chi\left(v^{\prime}\right) \equiv \chi(v)+w(\bmod n)$. The fact that $\operatorname{gcd}(n, w)=1$ also implies that $w$ is a generator of $\mathbb{Z}_{n}$, and so for every string $v \in S(n, w)$, its $n$ cyclic shifts are all distinctly colored. Thus, $S(n, w)$ has an equal number of strings from each color and the arguments used in the previous theorems will not rule out the existence of cyclic optimal codes.

\section{LOW-WEIGHT ANALYSis AND CONSTRUCTIONS}

In this section we study $(1,2, n ; w)$-LRMGCs having low weight, $w \leqslant 3$ (and by flipping bits and reversing strings, for all $w \geqslant n-3$ ). In the first trivial case of $w=1$, there exists a cyclic optimal code for all $n$. As we show in this section, the next two cases, namely $w=2,3$, behave radically different: We start with the case of $w=2$ in which we show a non-cyclic optimal code always exists, but when adding the requirement that the code be cyclic, no cyclic optimal codes exist and the efficiency of any cyclic code is asymptotically bounded from above by $\frac{3}{4}+o(1)$. In contrast, we will show that for $w=3$ we can construct cyclic asymptotically-optimal codes.

\section{A. The Case of $w=2$}

For the case of $w=2$ a brute-force approach will suffice. For all $n \geqslant 2$, let us define the graph $\mathcal{G}_{n}$ whose vertex set is $S(n, 2)$ and an edge $v \rightarrow v^{\prime}$ exists iff $v^{\prime}=\tau_{j}(v)$ for some $0 \leqslant j \leqslant n-1$.

Since by Theorem 6 cyclic optimal codes may only exist for odd $n$, let us restrict ourselves to that case only. We will, however, specify which results are also valid for even $n$. For convenience, we index the vertices in the following way: $v_{k, \ell}$, where $1 \leqslant k \leqslant(n-1) / 2$ and $0 \leqslant \ell \leqslant n-1$, denotes the vertex corresponding to the string having 1's in positions $\ell$ and $\ell+k$, where throughout the section we take the indices modulo $n$ where appropriate. We shall conveniently refer to the first index as the row index, and the second index as the column index.

Using this indexing method the graph $\mathcal{G}_{n}$ takes on a simple form for odd $n \geqslant 5$ (the case $n=3$ is more degenerate):
- A vertex of the form $v_{1, \ell}$ has a single outgoing edge to $v_{2, \ell}$.

- A vertex of the form $v_{k, \ell}, 1<k<(n-1) / 2$, has two outgoing edges to $v_{k+1, \ell}$ and $v_{k-1, \ell+1}$.

- A vertex of the form $v_{(n-1) / 2, \ell}$ has two outgoing edges to $v_{(n-3) / 2, \ell+1}$ and $v_{(n-1) / 2, \ell+(n+1) / 2}$.

It is now evident that there is a one-to-one correspondence between simple paths in $\mathcal{G}_{n}$ and Gray codes. A simple construction for an optimal code which is (in general) not cyclic is the following.

Construction 1. Let $n \geqslant 3$ be an odd integer. We construct the following code $G=g_{0}, g_{1}, \ldots, g_{N-1}$. We first set $g_{0}=v_{1,0}$, and then set $g_{i+1}$ as a function of $g_{i}=v_{k, \ell}$ according to the following rules:

- If $k$ is odd and $k<(n-1) / 2$, then $g_{i+1}=v_{k+1, \ell}$.

- If $k$ is odd and $k=(n-1) / 2$, then $g_{i+1}=v_{k, \ell+(n+1) / 2}$.

- If $k$ is even and $\ell<n-k / 2$, then $g_{i+1}=v_{k-1, \ell+1}$.

- If $k$ is even and $\ell=n-k / 2$, then $g_{i+1}=v_{k+1, \ell}$.

Theorem 10. The code from Construction 1 is an optimal $(1,2, n ; 2)$-LRMGC.

Proof: It is readily verifiable that the transitions involved in the construction are all valid. Furthermore, the construction is easily seen to first exhaust rows $2 t-1$ and $2 t$, where $t \geqslant 1$, by alternating between them, and then moving to rows $2 t+1$ and $2 t+2$. If the number of rows is even, this is enough to cover all the vertices. If the number of rows is odd, then the last row is covered by transitioning along the row. Since $\operatorname{gcd}((n+1) / 2, n)=1,(n+1) / 2$ is a generator of $\mathbb{Z}_{n}$ and the transitions along row $(n-1) / 2$ cover all of it.

An example of Construction 1 is shown in Figure 3. When $n=3,5$, Construction 1 results in a cyclic code (the case $n=5$ was given in Table I).

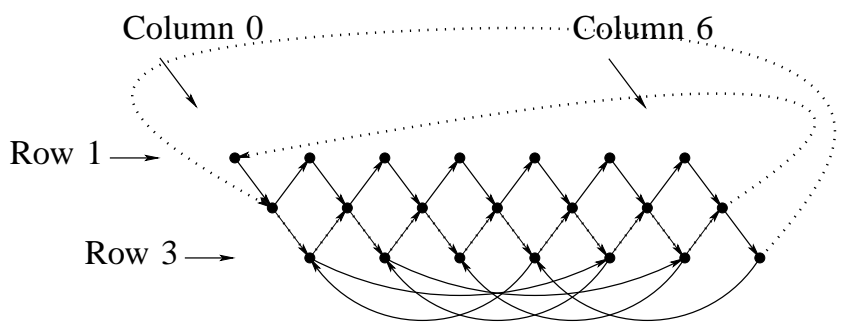

Figure 3. An example of an optimal non-cyclic $(1,2,7 ; 2)$-LRMGC which results from Construction 1. Solid arrows represent edges which are part of the code path, while dotted arrows represent those that are not.

Theorem 11. Let $G$ be a cyclic $(1,2, n ; 2)-L R M G C, n \geqslant 7$. Then $\operatorname{Eff}(G) \leqslant \frac{3}{4}+o(1)$.

Proof: We will prove the claim for odd $n$. The proof for even $n$ is essentially the same with a slight difference due to the different structure of the last row of $\mathcal{G}_{n}$. Let $G=$ $g_{0}, g_{1}, \ldots, g_{N-1}$ be cyclic Gray code, and let $v_{k_{i}, \ell_{i}}$ be the vertex corresponding to $g_{i}$. We say a vertex $v \in \mathcal{G}_{n}$ is covered if $v=v_{k_{i}, \ell_{i}}$ for some $0 \leqslant i \leqslant N-1$. We now denote by $k_{\min }$ and $k_{\max }$ the smallest and, respectively, largest, row index of vertices covered by the code $G$. 
The code obviously induces a cyclic path in $\mathcal{G}_{n}$, and therefore, there exist two sub-paths going "up" and "down" rows, $g_{u}, g_{u+1}, \ldots, g_{u^{\prime}}$ and $g_{d}, g_{d+1}, \ldots, g_{d^{\prime}}$, with the following properties: (indices are taken modulo $N$ where appropriate)

- $k_{u}=k_{\min }, k_{u^{\prime}}=k_{\max }$, and for all $0 \leqslant i \leqslant\left(u^{\prime}-\right.$ u) $\bmod N, k_{\min }<k_{u+i}<k_{\max }$.

- $k_{d}=k_{\max }, k_{d^{\prime}}=k_{\min }$, and for all $0 \leqslant i \leqslant\left(d^{\prime}-d\right) \bmod$ $N, k_{\min }<k_{d+i}<k_{\max }$.

The two sub-paths are obviously vertex disjoint, except perhaps the first and last vertices of the paths. Furthermore, one can easily be convinced, that the two paths do not occupy the same columns, except perhaps the columns of the first and last vertices of the paths. Along the "up" path, let $0 \leqslant t_{k_{\min }+1}, \ldots, t_{k_{\max }-1} \leqslant\left(u^{\prime}-u\right) \bmod N$ be the unique integers such that $g_{u+t_{i}}$ is the last vertex along the path at row $i$, i.e., $k_{u+t_{i}}=i$ and for all $t_{i}<j \leqslant\left(u^{\prime}-u\right) \bmod N$, $k_{u+j}>i$. It now follows that for each $k_{\min }<i<k_{\max }$, the vertices

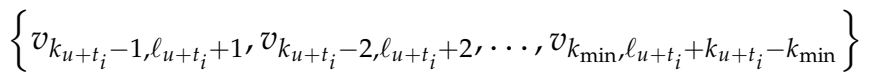

cannot be covered by any of the codewords of $G$. See an illustration in Figure 4 . The number of such uncovered vertices is exactly $\left(k_{\max }-k_{\min }\right)\left(k_{\max }-k_{\min }-1\right) / 2$.

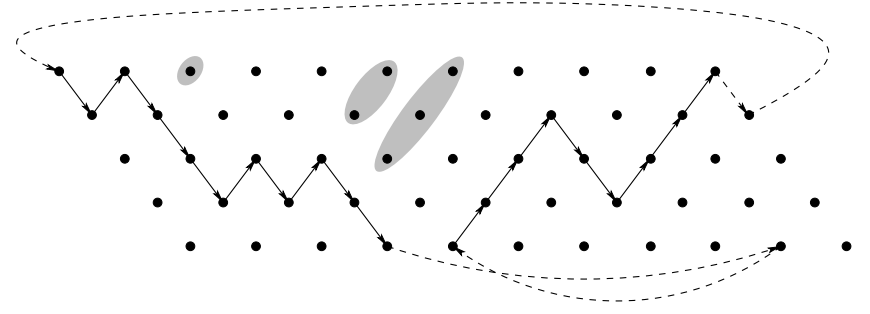

Figure 4. An example of a cyclic $(1,2,11 ; 2)$-LRMGC. Solid arrows represent edges which are part of the up and down paths, and the shaded vertices are those which are guaranteed to remain uncovered in the proof of Theorem 11.

In addition to the above-mentioned uncovered vertices, all the vertices of rows below $k_{\min }$ and above $k_{\max }$ are left uncovered by definition. Thus, if we denote $\delta=k_{\max }-k_{\min }$, the total number of uncovered vertices is at least

$$
n\left(\frac{n-1}{2}-\delta-1\right)+\frac{\delta(\delta-1)}{2} \geqslant \frac{1}{8}(n-3)(n-5),
$$

since the minimum is achieved at $\delta=\frac{n-3}{2}$. Therefore, the efficiency of the code $G$ is at most

$$
1-\frac{\frac{1}{8}(n-3)(n-5)}{\left(\begin{array}{c}
n \\
2
\end{array}\right)}=\frac{3}{4}+o(1)
$$

as claimed.

While the upper bound on the efficiency presented in Theorem 11 is $\frac{3}{4}+o(1)$, computer search results lead us to conjecture that it actually is $o(1)$.

\section{B. The Case of $w=3$}

In this section we turn to constructing asymptoticallyoptimal cyclic $(1,2, n ; 3)$-LRMGC. The construction will use a method originally used for constructing single-track Gray codes in [17] and later in [38]. In fact, the resulting codes will have the single-track property as well.

If $v=v_{0} v_{1} \ldots v_{n-1}$ is a length $n$ word over some alphabet, let $E$ denote the cyclic-shift operator defined by its action on v:

$$
E v=v_{n-1} v_{0} v_{1} \ldots v_{n-2} .
$$

The orbits under $E$ are called necklaces. A necklace is said to be full period if the smallest positive integer $i$ such that $E^{i} v=v$ is $i=n$. A full-period necklace contains $n$ distinct strings.

We say a Gray code $G=g_{0}, g_{1}, \ldots, g_{N-1}$ has the singletrack property if in the matrix whose $i$-th row is $g_{i}$, all the columns are cyclic shifts of each other. A variant of the following method was suggested in [17] for constructing single-track Gray codes, and it applies equally-well to our set of allowed transitions.

Lemma 12. Let $G^{\prime}=g_{0}^{\prime}, g_{1}^{\prime}, \ldots, g_{N^{\prime}-1}^{\prime}$ be a $(1,2, n ; w)$ LRMGC where $g_{i+1}^{\prime}=\tau_{j_{i}}\left(g_{i}^{\prime}\right)$ for all $0 \leqslant i \leqslant N^{\prime}-2$. If the strings in $G^{\prime}$ are representatives of distinct full-period necklaces, and $E^{\ell} g_{0}^{\prime}=\tau_{j_{N^{\prime}-1}} g_{N^{\prime}-1}^{\prime}, \operatorname{gcd}(\ell, n)=1$, then the following is a cyclic single-track Gray code:

$$
G=G^{\prime}, E^{\ell} G^{\prime}, E^{2 \ell} G^{\prime}, \ldots, E^{(n-1) \ell} G^{\prime},
$$

where $E^{j} G^{\prime}=E^{j} g_{0}^{\prime}, \ldots, E^{j} g_{N^{\prime}-1}^{\prime}$.

Proof: First, $E^{j} G^{\prime}$ is certainly also a Gray code. Since the necklaces in $G^{\prime}$ all have full cyclic period and since $\ell$ generates $\mathbb{Z}_{n}$, for $k \not \equiv k^{\prime}(\bmod n)$ the codes $E^{k \ell} G^{\prime}$ and $E^{k^{\prime} \ell} G^{\prime}$ are disjoint. Finally, it is easy to see that the transition from the last string of $E^{k \ell} G^{\prime}$ to the first string of $E^{(k+1) \ell} G^{\prime}$ is valid.

We define the mapping $\psi: S(n, 3) \rightarrow \mathbb{Z}_{n}^{3}$ as follows: for a binary string $v$ of length $n$ and weight 3 with 1 's in positions $0 \leqslant i_{0}<i_{1}<i_{2} \leqslant n-1$, let

$$
\psi(v)=\left(i_{1}-i_{0}, i_{2}-i_{1}, i_{0}-i_{2}\right)
$$

where subtraction is made modulo $n$. The set $\{\psi(v) \mid v \in S(n, 3)\}$ is the set of points $\left(d_{0}, d_{1}, d_{2}\right) \in \mathbb{Z}^{3}$ that are on the hyperplane $d_{0}+d_{1}+d_{2}=n$ restricted to $1 \leqslant d_{0}, d_{1}, d_{2} \leqslant n-2$. We call $\psi(v)$ the configuration of $v$. We note that if $\operatorname{gcd}(n, 3)=1$, then $S(n, 3)$ contains only full-period strings, and otherwise, all strings are full-period except those with configuration $(n / 3, n / 3, n / 3)$. We denote by $S^{*}(n, 3)$ the set of full-period strings from $S(n, 3)$.

Since $\psi(v), E \psi(v)$, and $E^{2} \psi(v)$, (corresponding to a cyclic rotation of the axes of $\left.\mathbb{Z}^{3}\right)$, represent strings from the same necklace, for any $v \in S^{*}(n, 3)$, let $\psi^{\prime}(v)$ stand for the unique $\left(d_{0}, d_{1}, d_{2}\right) \in\left\{\psi(v), E \psi(v), E^{2} \psi(v)\right\}$ for which $d_{1} \leqslant\lfloor n / 3\rfloor$ and $d_{2}>\lfloor n / 3\rfloor$. Thus, there is a simple one-to-one mapping from $\left\{\psi^{\prime}(v) \mid v \in S^{*}(n, 3)\right\}$ to the set of full-period necklaces. We call $\psi^{\prime}(v)$ the canonical configuration of $v$.

A simple counting reveals that there are a total of $\frac{(n-1)(n-2)}{2}$ configurations, and when $\operatorname{gcd}(n, 3)=1$ there are $\frac{(n-1)(n-2)}{6}=\frac{1}{n}\left(\begin{array}{l}n \\ 3\end{array}\right)$ canonical configurations which is exactly the number of weight- 3 full-period necklaces. When $\operatorname{gcd}(n, 3) \neq 1$, there are $\frac{(n-1)(n-2)-2}{6}$ canonical configurations. See Figure 5 for an illustration. 


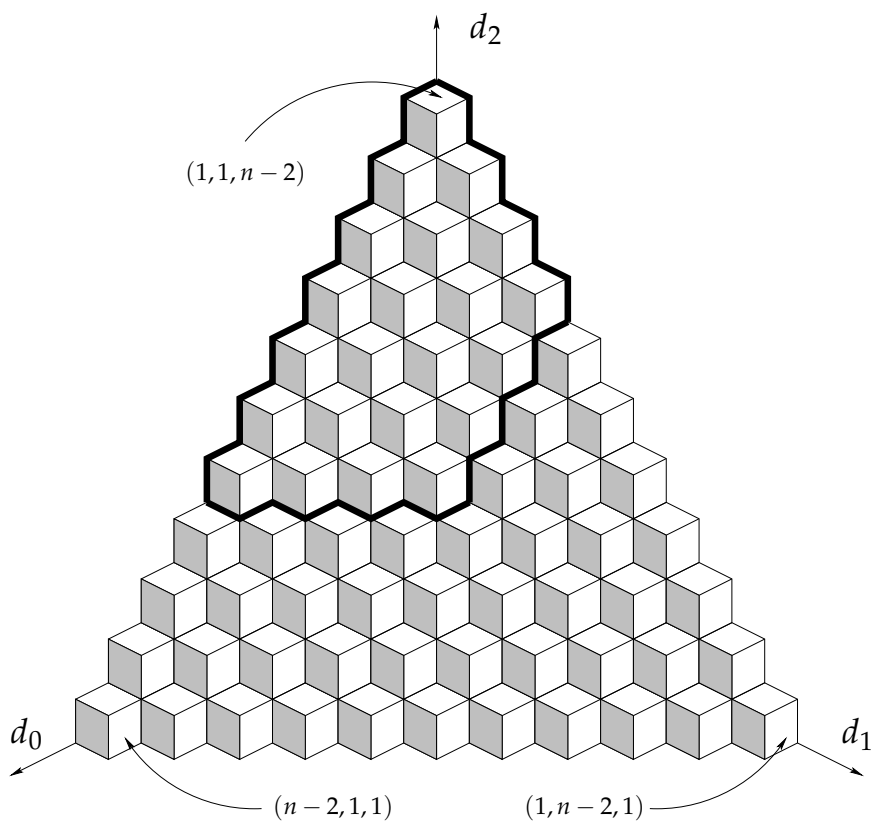

Figure 5. The hyperplane of configurations for $n=13$. The set of canonical configurations is shown surrounded by a thick frame.

Lemma 13. Let $\Delta=\left(d_{0}, d_{1}, d_{2}\right)$ be a canonical configuration, and assume

$$
\begin{aligned}
\Delta^{\prime} \in\{ & \left(d_{0}+1, d_{1}-1, d_{2}\right), \\
& \left(d_{0}, d_{1}+1, d_{2}-1\right), \\
& \left.\left(d_{0}-1, d_{1}, d_{2}+1\right)\right\}
\end{aligned}
$$

is also a canonical configuration. Then for any $v \in S^{*}(n, 3)$ such that $\psi^{\prime}(v)=\Delta$ there exists $v^{\prime} \in S^{*}(n, 3)$ such that $\psi^{\prime}\left(v^{\prime}\right)=\Delta^{\prime}$ and $v^{\prime}=\tau_{j}(v)$ for some $0 \leqslant j \leqslant n-1$.

Proof: Assume $\Delta^{\prime}=\left(d_{0}+1, d_{1}-1, d_{2}\right)$ is a canonical configuration (the proof for the two other cases is similar). Let $v \in S^{*}(n, 3)$ be such that $\psi^{\prime}(v)=\Delta$, i.e., there exists some $0 \leqslant i \leqslant n-1$ such that the 1 's in $v$ occur in positions $i, i+d_{0}$, and $i+d_{0}+d_{1}$ (all taken modulo $n$ ). It is easily verified that $v^{\prime}=\tau_{i+d_{0}}(v)$ has canonical configuration $\Delta^{\prime}$.

We now intend to find a long cycle over canonical configurations which, by Lemma 13, will result in a Gray code of representatives of distinct full-period necklaces. The latter will be used with Lemma 12 to generate a cyclic $(1,2, n ; 3)$ LRMGC.

Construction 2. Let $n \geqslant 9$ be an integer. We construct the following sequence of canonical configurations $\Gamma=$ $\Delta_{0}, \Delta_{1}, \ldots, \Delta_{N^{\prime}-1}$. We first set $\Delta_{0}=(1,1, n-2)$, and then set $\Delta_{i+1}$ as a function of $\Delta_{i}=\left(d_{0}, d_{1}, d_{2}\right)$ according to the following rules:

- If $d_{0}=1$ and $d_{1}<3\lfloor\lfloor n / 3\rfloor / 3\rfloor$, then set $\Delta_{i+1}=$ $\left(d_{0}, d_{1}+1, d_{2}-1\right)$.

- Else, if $d_{1} \equiv 0(\bmod 3)$, then set $\Delta_{i+1}=\left(d_{0}+1, d_{1}-\right.$ $\left.1, d_{2}\right)$.

- Else, if $d_{1} \equiv 2(\bmod 3)$ and $d_{2}>\lfloor n / 3\rfloor+1$, then set $\Delta_{i+1}=\left(d_{0}, d_{1}+1, d_{2}-1\right)$.

- Else, if $d_{1} \equiv 2(\bmod 3)$ and $d_{2}=\lfloor n / 3\rfloor+1$ and $d_{1}>$ 1 , then set $\Delta_{i+1}=\left(d_{0}+1, d_{1}-1, d_{2}\right)$.
- Else, if $d_{1} \equiv 1(\bmod 3)$ and $d_{0}>2$, then set $\Delta_{i+1}=$ $\left(d_{0}-1, d_{1}, d_{2}+1\right)$.

- To complete the cycle, if $\Delta_{i}=(1,2, n-3)$, then set $\Delta_{i+1}=(1,1, n-2)$.

An illustration of the path from Construction 2 is shown in Figure 6.

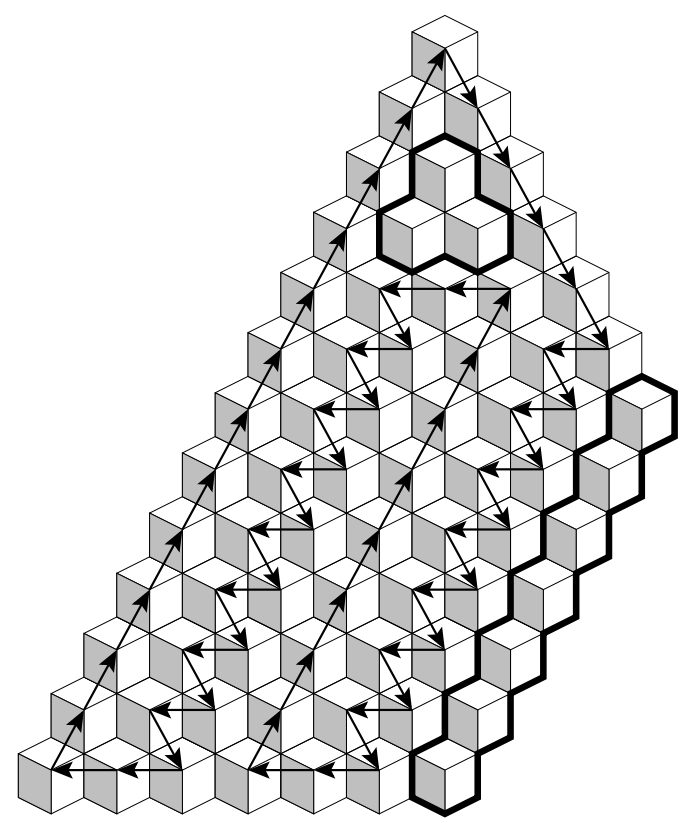

Figure 6. The path from Construction 2 over the canonical configurations for $n=22$. The unvisited configurations are shown surrounded by a thick frame.

Lemma 14. The path from Construction 2 visits only canonical configurations, each visited no more than once.

Proof: Going over all the transitions in Construction 2 one can verify that they visit only canonical configurations. Except for configurations of the form $\left(1, d_{1}, d_{2}\right)$ which are part of path of increasing $d_{1}$, the rest of the path is divided according to $d_{1} \bmod 3:$ when $d_{1} \equiv 2,0(\bmod 3)$ the path zigzags "downward", and goes back "up" when $d_{1} \equiv 1(\bmod 3)$ (see Figure 6). This path structure ensures no vertex is visited more than once in a cycle.

Lemma 15. The length $N^{\prime}$ of the path from Construction 2 is given by

$$
N^{\prime}(n)=\left\{\begin{array}{lll}
\frac{n^{2}-5 n+18}{6} & n \equiv 0 & (\bmod 9) \\
\frac{n^{2}-5 n+22}{6} & n \equiv 1 & (\bmod 9) \\
\frac{n^{2}-5 n+24}{6} & n \equiv 2 & (\bmod 9) \\
\frac{n^{2}-7 n+30}{6} & n \equiv 3 & (\bmod 9) \\
\frac{n^{2}-7 n+30}{6} & n \equiv 4 & (\bmod 9) \\
\frac{n^{2}-7 n+28}{6} & n \equiv 5 & (\bmod 9) \\
\frac{n^{2}-9 n+36}{6} & n \equiv 6 & (\bmod 9) \\
\frac{n^{2}-9 n+32}{6} & n \equiv 7 & (\bmod 9) \\
\frac{n^{2}-9 n+26}{6} & n \equiv 8 & (\bmod 9)
\end{array}\right.
$$


Proof: The path length depends on the number of times it zigzags "downward" which is $\lfloor\lfloor n / 3\rfloor / 3\rfloor$. The rest is a careful and tedious counting.

Lemma 16. Let $G^{\prime}=g_{0}^{\prime}, g_{1}^{\prime}, \ldots, g_{N^{\prime}-1}^{\prime}$ be a list of strings from $S^{*}(n, 3)$ (whose existence is guaranteed by Lemma 13) such that $\Gamma=\psi^{\prime}\left(g_{0}^{\prime}\right), \psi^{\prime}\left(g_{1}^{\prime}\right), \ldots, \psi^{\prime}\left(g_{N^{\prime}-1}^{\prime}\right)$ is the cyclic path from Construction 2. Let $g^{*}$ be the string (whose existence is guaranteed by Lemma 13) such that $\psi^{\prime}\left(g^{*}\right)=\psi^{\prime}\left(g_{0}^{\prime}\right)$ and $g^{*}=\tau_{j}\left(g_{N^{\prime}-1}^{\prime}\right)$. Then $g^{*}=E^{N^{\prime} / 3} g_{0}^{\prime}$.

Proof: Let us examine $g_{i}^{\prime}$ for some $i$ and suppose we could distinguish between the three 1's in $g_{i}^{\prime}$ by coloring them red, blue, and green. If $\psi^{\prime}\left(g_{i}^{\prime}\right)=\left(d_{0}, d_{1}, d_{2}\right)$, assume w.l.o.g., that $d_{0}$ is the distance between the red and blue 1's, $d_{1}$ between the blue and green 1's, and $d_{2}$ between the green and red 1's. If $\psi^{\prime}\left(g_{i+1}^{\prime}\right)=\left(d_{0}^{\prime}, d_{1}^{\prime}, d_{2}^{\prime}\right)$, then a careful reading of Lemma 13 shows that in $g_{i+1}^{\prime}, d_{0}^{\prime}$ is again the distance between the red and blue 1's, $d_{1}^{\prime}$ between the blue and green 1 's, and $d_{2}^{\prime}$ between the green and red 1's.

Since $\psi^{\prime}\left(g^{*}\right)=\psi^{\prime}\left(g_{0}^{\prime}\right)$ it follows that $g^{*}$ is a cyclic shift of $g_{0}^{\prime}$. By the previous argument, to get from $g_{0}^{\prime}$ to $g^{*}$, all the 1 's had to be pushed an equal number of times to the right and so $g^{*}=E^{N^{\prime} / 3} g_{0}^{\prime}$.

The following is the main theorem of this section:

Theorem 17. For all $n \geqslant 9$ such that $\operatorname{gcd}\left(n, N^{\prime}(n) / 3\right)=1$, where $N^{\prime}(n)$ is given by $(5)$, there exists a cyclic $(1,2, n ; 3)$ LRMGC of size $N=n \cdot N^{\prime}(n)$, which is also single-track.

Proof: By Lemma 13, let $G^{\prime}=g_{0}^{\prime}, g_{1}^{\prime}, \ldots, g_{N^{\prime}-1}^{\prime}$ be a list of strings from $S^{*}(n, 3)$ such that $\Gamma=$ $\psi^{\prime}\left(g_{0}^{\prime}\right), \psi^{\prime}\left(g_{1}^{\prime}\right), \ldots, \psi^{\prime}\left(g_{N^{\prime}-1}^{\prime}\right)$ is the cyclic path from Construction 2. By Lemma 15, $N^{\prime}=N^{\prime}(n)$ from (5). According to Lemma 14, $\Gamma$ contains distinct canonical configurations, and so $G^{\prime}$ contains representatives of distinct full-period necklaces. Finally, by combining Lemma 16 with the requirement that $\operatorname{gcd}\left(n, N^{\prime}(n) / 3\right)=1$, we can use Lemma 12 to construct the desired code.

Lemma 18. There are infinite values of $n \in \mathbb{N}$ for which $\operatorname{gcd}\left(n, N^{\prime}(n) / 3\right)=1$. More specifically, it suffices that $n$ satisfies one of the following:

- $n \equiv 7,11(\bmod 18)$

- $n \equiv 13,31,49,67(\bmod 90)$

- $n \equiv 5,23,41,59,95,113(\bmod 126)$

- $n \equiv 1,19,37,73,91,109,127,145,163,181(\bmod 198)$

- $n \equiv 17,35,53,71,89,107,125,161,179,197,215,233$

$(\bmod 234)$

Proof: We will prove one of the cases and the rest are similar. Assume $n \equiv 4(\bmod 9)$. By Lemma 15 we need

$$
\operatorname{gcd}\left(n, \frac{n^{2}-7 n+30}{18}\right)=1
$$

Since $\operatorname{gcd}(a, b)$ divides any integer combination of $a$ and $b$, and since

$$
18 \cdot \frac{n^{2}-7 n+30}{18}-(n-7) \cdot n=30
$$

it follows that

$$
\operatorname{gcd}\left(n, \frac{n^{2}-7 n+30}{18}\right) \mid 30
$$

Thus, if we could only make sure that $\operatorname{gcd}(n, 30)=1$ the claim would necessarily follow. Combining $\operatorname{gcd}(n, 30)=$ 1 and $n \equiv 4(\bmod 9)$, we get that $n \equiv 13,31,49,67$ (mod 90) is sufficient to prove the claim.

We note that the conditions described in Lemma 18 are not the only cases in which $\operatorname{gcd}\left(n, N^{\prime}(n) / 3\right)=1$, but are just the ones easy to derive. For instance, when $n=27$, we have $\operatorname{gcd}\left(n, N^{\prime}(n) / 3\right)=\operatorname{gcd}(27,34)=1$.

Corollary 19. There exists an infinite family $\left\{G_{i}\right\}_{i=1}^{\infty}$ of cyclic $\left(1,2, n_{i} ; 3\right)$-LRMGCs, $n_{i+1}>n_{i}$ for all $i$, for which $\lim _{i \rightarrow \infty} \operatorname{Eff}\left(G_{i}\right)=1$.

Proof: Simply combine Lemma 18 with the fact that

$$
\lim _{n \rightarrow \infty} \frac{n \cdot N^{\prime}(n)}{\left(\begin{array}{l}
n \\
3
\end{array}\right)}=1
$$

On a final note, the codes from Theorem 17 turn out to be optimal in the cases of $n=10,11$ with sizes $N=120,165$ respectively.

\section{Asymptotically Constant Rate Codes}

The main problem with the codes constructed in Section IV is that their rate is asymptotically 0 . We now turn to constructing codes with rates asymptotically tending to 1 , and weight asymptotically half the length, thus having asymptoticallyoptimal charge difference between adjacent cells.

Our construction has the following intuitive flavor. We start by partitioning the $n$ flash cells into about $\sqrt{n}$ blocks, each block of size about $\sqrt{n}$, treating each block of cells as a single character in a large alphabet, say $\{0,1, \ldots, t-1\}$ for $t \simeq 2^{\sqrt{n}}$. Roughly speaking, by this operation, we have reduced the problem of finding a Gray code over $\{0,1\}^{n}$ into an outer Gray-like code over $\{0,1, \ldots, t-1\}^{\sqrt{n}}$. Several Gray codes of rate 1 exist over large alphabets, however, not any outer code will suffice in our setting. Primarily, it is crucial that we may move from state to state in the outer code using our elementary pairwise "push-to-the-top" operations. Moreover, in doing so, we must guarantee that flash cell values obtained between a single representation of the outer codeword and its successor are unique. We achieve these goals using an outer Gray code based on de-Bruijn sequences. In such codes, the location of the character that changes between subsequent codewords over goes a cyclic shift. This cyclic location change between subsequent codewords lends itself very naturally to our cyclic "push-to-the-top" operations. Combining this with additional ideas, that guarantee distinct cell values (of constant weight) in transition between outer codewords, we obtain our construction.

Construction 3. Fix a positive integer $k$. Let $\left\{v_{0}, v_{1}, \ldots, v_{t-1}\right\}$ be a set of $t$ distinct binary vectors of length $m+2$ and weight $w+2$ such that the first and last bit of each $v_{i}$ is 1 . We also denote $L=\operatorname{lcm}\left(k+2, t^{k}\right)$. 
The next required ingredient in the construction is a deBruijn sequence of order $k$ over the alphabet $\{0,1, \ldots, t-1\}$. The sequence is of period $t^{k}$ and we denote it by $s_{0}, s_{1}, \ldots, s_{t^{k}-1}$. We remind the reader that windows of size $k$ in the sequence, i.e., $s_{i}, s_{i+1}, \ldots, s_{i+k-1}$, with indices taken modulo $t^{k}$, are all distinct. Such sequences can always be constructed (for example, see [21]).

We now construct the sequence $g_{0}, g_{1}, \ldots, g_{L-1}$ of $L$ binary vectors of length $(k+2)(m+2)$ and weight $(k+1)(w+2)$. Each vector is formed by a concatenation of $k+2$ blocks of length $m+2$ in the following way:

$$
\begin{aligned}
& \begin{array}{lllllll}
g_{0}= & v_{s_{k}} & v_{s_{k-1}} & \ldots & v_{s_{1}} & v_{s_{0}} & \mathbf{0}
\end{array}
\end{aligned}
$$

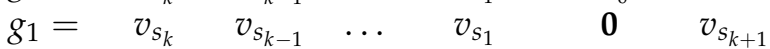



$$
\begin{aligned}
& \begin{array}{lllllll}
g_{k}= & v_{s_{k}} & \mathbf{0} & \ldots & v_{s_{k+3}} & v_{s_{k+2}} & v_{s_{k+1}}
\end{array}
\end{aligned}
$$

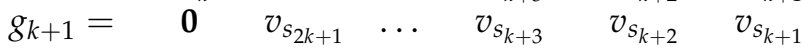

$$
\begin{aligned}
& \begin{array}{lllllll}
g_{k+2}= & v_{s_{2 k+2}} & v_{s_{2 k+1}} & \cdots & v_{s_{k+3}} & v_{s_{k+2}} & \mathbf{0}
\end{array} \\
& \begin{array}{lllllll}
g_{k+3} & =v_{s_{2 k+2}} & v_{s_{2 k+1}} & \cdots & v_{s_{k+3}} & \mathbf{0} & v_{s_{2 k+3}}
\end{array} \\
& \begin{array}{lllllll}
g_{k+4} & =v_{s_{2 k+2}} & v_{s_{2 k+1}} & \cdots & \mathbf{0} & v_{s_{2 k+4}} & v_{s_{2 k+3}}
\end{array} \\
& \begin{array}{lllllll}
g_{2 k+2} & =v_{S_{2 k+2}} & \mathbf{0} & \ldots & v_{S_{2 k+5}} & v_{S_{2 k+4}} & v_{S_{2 k+3}}
\end{array} \\
& g_{2 k+3}=\begin{array}{lllllll}
\mathbf{0} & v_{S_{3 k+3}} & \cdots & v_{S_{2 k+5}} & v_{S_{2 k+4}} & v_{S_{2 k+3}}
\end{array}
\end{aligned}
$$

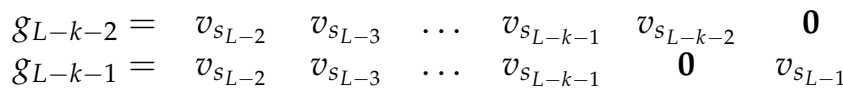

$$
\begin{aligned}
& \begin{array}{lllllll}
g_{L-k} & =v_{s_{L-2}} & v_{S_{L-3}} & \ldots & \mathbf{0} & v_{s_{0}} & v_{s_{L-1}}
\end{array} \\
& \begin{array}{ccccccc}
g_{L-2} & =v_{S_{L-2}} & \mathbf{0} & \ldots & v_{S_{1}} & v_{S_{0}} & v_{S_{L-1}} \\
g_{L-1}= & \mathbf{0} & v_{S_{k-1}} & \ldots & v_{S_{1}} & v_{s_{0}} & v_{S_{L-1}}
\end{array}
\end{aligned}
$$

where 0 denotes the all-zero vector of length $m+2$, and the sub-indices of $s$ are taken modulo $t^{k}$.

We call $g_{0}, g_{1}, \ldots, g_{L-1}$ the anchor vectors. We note that between anchors $g_{i}$ and $g_{i+1}$ the block $v_{s_{i}}$ moves $m+2$ positions to the right (with wrap-around) and is changed to the block $v_{s_{i+k+1}}$.

Finally, between any two anchors, $g_{i}$ and $g_{i+1}$, a sequence of vectors called auxiliary vectors and denoted $g_{i}^{0}, g_{i}^{1}, \ldots, g_{i}^{\ell_{i}}$, is formed in the following way: The only allowed transition is a 10 changed into a 01 . First the rightmost 1 in the block $v_{s_{i}}$ is moved to the right, step by step, to the position of the rightmost 1 in $v_{s_{i+k+1}}$. The process then repeats with a sequence of transitions moving the second-from-right 1 in $v_{s_{i}}$ to the position of the second-from-right 1 in $v_{s_{i+k+1}}$, and so on, until $v_{s_{i}}$ is moved one block to the right and changed into $v_{s_{i+k+1}}$ (see Example 20). The resulting list of anchor vectors and, in between them, auxiliary vectors, is the constructed code.

Example 20. Let us take a very simple case of $k=1, m=3$, $w=2$, and $t=3$, with $s_{0}=0, s_{1}=1$, and $s_{2}=2$, and then

\begin{tabular}{|c|c|c|c|}
\hline & & & \\
\hline $\begin{array}{l}0 \\
0=\end{array}$ & 11011 & $0 \underline{0}$ & 000 \\
\hline & 11011 & 11100 & $\underline{01000}$ \\
\hline & 11011 & 11100 & $\overline{00100}$ \\
\hline & 11011 & 11100 & 0001 \\
\hline & 11011 & 11100 & $000 \underline{0}$ \\
\hline & 11011 & $11 \underline{010}$ & 00001 \\
\hline & 11011 & $\underline{11}$ & 0000 \\
\hline & 11011 & $\underline{0}$ & \\
\hline & 011 & 10 & $\overline{01}$ \\
\hline & 1 & 110 & $\overline{0 \underline{0}}$ \\
\hline & 11011 & 11000 & $0 \overline{00 \underline{1}}$ \\
\hline & 11011 & $1 \underline{0100}$ & 00011 \\
\hline & 11011 & $10 \underline{010}$ & 00011 \\
\hline & 11011 & $10 \overline{001}$ & 0001 \\
\hline & 11011 & 10 & \\
\hline & 11011 & 10 & $\underline{0101]}$ \\
\hline & 11011 & 100 & $0 \underline{011}$ \\
\hline & 11011 & $\underline{01000}$ & $0 \overline{011}$ \\
\hline & 11011 & $\overline{001} 100$ & 00 \\
\hline & 11011 & $0 \overline{001} 0$ & 001 \\
\hline & 011 & 000 & \\
\hline & & 000 & \\
\hline
\end{tabular}
$v_{0}=11101, v_{1}=11011$, and $v_{2}=10111$. The list of anchors is

$$
\begin{aligned}
& g_{0}=11011 \quad 1110100000 \\
& g_{1}=110110000010111 \\
& g_{2}=00000 \quad 11101 \quad 10111
\end{aligned}
$$

and, for example, the transition between $g_{0}$ and $g_{1}$ is (the changed positions are underlined)

Theorem 21. The code constructed in Construction 3 is a cyclic $(1,2,(k+2)(m+2) ;(k+1)(w+2))-L R M G C$ of size

$N=L(w+2)(m+2)=\operatorname{lcm}\left(t^{k}, k+2\right) \cdot(w+2) \cdot(m+2)$.

Proof: That the code contains only valid transitions is evident by the construction method. We need to show that all the constructed codewords are distinct which we do with the following reasoning: consider some constructed codeword $g$ of length $(k+2)(m+2)$ and weight $(k+1)(w+2)$. Deciding whether $g$ is an anchor is simple, since only anchors have $k+1$ blocks beginning and ending with a 1 , and the remaining block a $\mathbf{0}$. By our choice of $L$, all anchors are distinct since they contain windows of size $k+1$ from a de-Bruijn sequence of order $k$, each window appearing in $(k+2) / \operatorname{gcd}\left(k+2, t^{k}\right)$ distinct cyclic shifts (which are easily distinguishable by the position of the $\mathbf{0}$ block). It then follows that if $g$ is indeed an anchor it appears only once in the code.

Assume we discover $g$ is an auxiliary vector. Again, by construction, all auxiliary vectors between $g_{i}$ and $g_{i+1}$ have $k$ fixed blocks. Looking at $g$, an auxiliary vector, exactly $k$ blocks are of weight $w+2$ while the other two blocks have weight strictly below $w+2$. The blocks of weight $w+2$, by construction, form a window of size $k$ from a de-Bruijn sequence of order $k$ starting at $s_{i}$, and so their content and position uniquely identify between which two anchors $g$ lies.

Finally, all the auxiliary vectors between adjacent anchors $g_{i}$ and $g_{i+1}$ are easily seen to be distinct. Thus, given a codeword $g$ from the constructed code, there is exactly one position in the sequence of generated codewords which equals $g$, and so all generated codewords are distinct. 
To complete the proof we need to calculate the size $N$. There are exactly $L$ anchors. Given an anchor $g_{i}$, the number of steps in the transition to $g_{i+1}$ may be readily verified to be $(w+2)(m+2)+\chi\left(g_{i+1}\right)-\chi\left(g_{i}\right)$, where $\chi(\cdot)$ is the first moment function defined in (2). Thus,

$$
\begin{aligned}
N & =\sum_{i=0}^{L-1}\left((w+2)(m+2)+\chi\left(g_{i+1}\right)-\chi\left(g_{i}\right)\right) \\
& =L(w+2)(m+2)
\end{aligned}
$$

as claimed. As a final note, the choice of $L$ is easily seen to ensure the resulting code is cyclic.

We mention in passing that the proof of Theorem 21 hints at efficient encoding and decoding procedures, provided other efficient encoding and decoding procedures exist for de-Bruijn sequences. Examples of such procedures may be found in [33], [42].

We now turn to show the main claim of the section.

Corollary 22. There exists an infinite family $\left\{G_{i}\right\}_{i=1}^{\infty}$ of cyclic $\left(1,2, n_{i} ; w_{i}\right)$-LRMGCs, $n_{i+1}>n_{i}$ for all $i$, for which $\lim _{i \rightarrow \infty} \mathrm{R}\left(G_{i}\right)=1$, and $\lim _{i \rightarrow \infty} \frac{w_{i}}{n_{i}}=\frac{1}{2}$.

Proof: For the code $G_{i}$, set $w=i$, and $k=m=2 i$ (i.e., $n_{i}=(2 i+2)^{2}$ and $\left.w_{i}=(2 i+1)(i+2)\right)$ and apply Theorem 21 with $t=\left(\begin{array}{c}2 i \\ i\end{array}\right)$. The size, $N_{i}$, of the code $G_{i}$, is bounded by

$$
\left(\begin{array}{c}
2 i \\
i
\end{array}\right)^{2 i}(i+2)(2 i+2) \leqslant N_{i} \leqslant\left(\begin{array}{c}
2 i \\
i
\end{array}\right)^{2 i}(i+2)(2 i+2)^{2}
$$

since

$$
\left(\begin{array}{c}
2 i \\
i
\end{array}\right)^{2 i} \leqslant \operatorname{lcm}\left(\left(\begin{array}{c}
2 i \\
i
\end{array}\right)^{2 i}, 2 i+2\right) \leqslant\left(\begin{array}{c}
2 i \\
i
\end{array}\right)^{2 i}(2 i+2) .
$$

It is well known (see for example [32], p. 309) that for any $0<\lambda<1$, assuming $\lambda \ell$ is an integer,

$$
\frac{1}{\sqrt{8 \ell \lambda(1-\lambda)}} 2^{\ell H(\lambda)} \leqslant\left(\begin{array}{c}
\ell \\
\lambda \ell
\end{array}\right) \leqslant \frac{1}{\sqrt{2 \ell \lambda(1-\lambda)}} 2^{\ell H(\lambda)}
$$

where $H(\cdot)$ is the binary entropy function. Since $H(1 / 2)=1$, it now easily follows that

$$
\lim _{i \rightarrow \infty} \mathrm{R}\left(G_{i}\right)=1, \quad \quad \lim _{i \rightarrow \infty} \frac{w_{i}}{n_{i}}=\frac{1}{2} .
$$

If needed, we can achieve lower asymptotic rates by setting $w=\lambda m$ for some rational $0<\lambda<1, \lambda \neq 1 / 2$.

\section{CONCLUSION}

We presented the general framework of $(s, t, n)$-local rank modulation and focused on the specific case of $(1,2, n)$-LRM which is both the least-hardware-intensive, and the simplest one to translate between binary strings and permutations. We studied constant-weight Gray codes for this scheme, which guarantee a bounded charge difference in any "push-to-thetop" operation. The Gray codes are used to simulate a conventional multi-level flash cell.

Using coloring and counting arguments we derived necessary conditions for the existence of cyclic and cyclic optimal $(1,2, n ; w)$-LRMGCs.
While cyclic optimal Gray codes exist (trivially) for $w=1$, we showed that for $w=2$ their efficiency is upper bounded by $\frac{3}{4}+o(1)$. In contrast, for $w=3$ asymptotically-optimal codes exist with efficiency $1-o(1)$. The codes we constructed also come with a relatively simple updating algorithm. Finally, by letting $w$ be approximately $n / 2$ we constructed cyclic $(1,2, n ; w)$-LRMGCs whose rate approaches 1 .

Several open questions still remain. For the case of $(1,2, n ; w)$-LRMGCs, a general construction is missing for constant weights $w \geqslant 4$. We also conjecture, based on computer search results, that for $w=2$ and $n$ large enough, the size of cyclic codes is at most $2 n$, hence, with efficiency actually $o(1)$. Of more general interest is the study of codes for general $(s, t, n)$-LRM and their parameters.

\section{REFERENCES}

[1] T. M. Apostol, Introduction to Analytic Number Theory. SpringerVerlag, NY, 1976.

[2] T. Berger, F. Jelinek, and J. K. Wolf, "Permutation codes for sources," IEEE Trans. on Inform. Theory, vol. IT-18, no. 1, pp. 160-169, Jan. 1972.

[3] I. F. Blake, "Permutation codes for discrete channels," IEEE Trans. on Inform. Theory, vol. 20, pp. 138-140, 1974.

[4] I. F. Blake, G. Cohen, and M. Deza, "Coding with permutations," Inform. and Control, vol. 43, pp. 1-19, 1979.

[5] V. Bohossian, A. Jiang, and J. Bruck, "Buffer coding for asymmetric multi-level memory," in Proceedings of the 2007 IEEE International Symposium on Information Theory (ISIT2007), Nice, France, Jun. 2007, pp. 1186-1190.

[6] M. Buck and D. Wiedemann, "Gray codes with restricted density," Discrete Math., vol. 48, pp. 163-181, 1984.

[7] M. Carkeet and P. Eades, "A subset generation algorithm with a very strong minimal change property," Congressus Numerantium, vol. 47, pp. 139-143, 1985.

[8] H. Chadwick and I. Reed, "The equivalence of rank permutation codes to a new class of binary codes," IEEE Trans. on Inform. Theory, vol. 16, no. 5, pp. 640-641, 1970.

[9] H. D. Chadwick and L. Kurz, "Rank permutation group codes based on Kendall's correlation statistic," IEEE Trans. on Inform. Theory, vol. IT-15, no. 2, pp. 306-315, Mar. 1969.

[10] C. C. Chang, H. Y. Chen, and C. Y. Chen, "Symbolic Gray code as a data allocation scheme for two-disc systems," Comput. J., vol. 35, pp. 299-305, 1992.

[11] M. Chen and K. G. Shin, "Subcube allocation and task migration in hypercube machines," IEEE Trans. on Comput., vol. 39, pp. 1146-1155, 1990.

[12] G. Cohen and M. Deza, "Decoding of permutation codes," in Intl. CNRS Colloquium, July, France, 1977.

[13] M. Deza and P. Frankl, "On maximal numbers of permutations with given maximal or minimal distance," J. Combin. Theory Ser. A, vol. 22, 1977.

[14] P. Diaconis and S. Holmes, "Gray codes for randomization procedures," Stat. Comput., vol. 4, pp. 287-302, 1994.

[15] L. E. Dickson, History of the Theory of Numbers, vol. 2. New York: Chelsea, 1952.

[16] P. Eades, M. Hickey, and R. C. Read, "Some Hamiltonian paths and minimal change algorithms," J. of the ACM, vol. 31, pp. 19-29, 1984.

[17] T. Etzion and K. G. Paterson, "Near optimal single-track Gray codes," IEEE Trans. on Inform. Theory, vol. 42, no. 3, pp. 779-789, May 1996.

[18] C. Faloutsos, "Gray codes for partial match and range queries," IEEE Trans. on Software Eng., vol. 14, pp. 1381-1393, 1988.

[19] H. C. Ferriera, A. J. H. Vinck, T. G. Swart, and I. de Beer, "Permutation trellis codes," IEEE Trans. on Communications, pp. 1782-1789, Nov. 2005.

[20] M. Gardner, "The curious properties of the Gray code and how it can be used to solve puzzles," Scientif. Amer., vol. 227, pp. 106-109, 1972.

[21] S. W. Golomb, Shift Register Sequences. Holden-Day, San Francisco, 1967.

[22] F. Gray, "Pulse code communication," March 1953, U.S. Patent 2632058. 
[23] T. Hough and F. Ruskey, "An efficient implementation of the Eades, Hickey, Read adjacent interchange combination generation algorithm," J. of Comb. Math. and Comb. Comp., vol. 4, pp. 79-86, 1988.

[24] A. Jiang, V. Bohossian, and J. Bruck, "Floating codes for joint information storage in write asymmetric memories," in Proceedings of the 2007 IEEE International Symposium on Information Theory (ISIT2007), Nice, France, Jun. 2007, pp. 1166-1170.

[25] A. Jiang and J. Bruck, "Joint coding for flash memory storage," in Proceedings of the 2008 IEEE International Symposium on Information Theory (ISIT2008), Toronto, Canada, Jul. 2008, pp. 1741-1745.

[26] A. Jiang, M. Langberg, M. Schwartz, and J. Bruck, "Universal rewriting in constrained memories," in Proceedings of the 2009 IEEE International Symposium on Information Theory (ISIT2009), Seoul, Korea, Jun. 2009, pp. 1219-1223.

[27] A. Jiang, R. Mateescu, M. Schwartz, and J. Bruck, "Rank modulation for flash memories," IEEE Trans. on Inform. Theory, vol. 55, no. 6, pp. 2659-2673, Jun. 2009.

[28] A. Jiang, M. Schwartz, and J. Bruck, "Correcting charge-constrained errors in the rank-modulation scheme," IEEE Trans. on Inform. Theory, vol. 56, no. 5, pp. 2112-2120, May 2010.

[29] C. A. Laisant, "Sur la numération factorielle, application aux permutations," Bulletin de la Société Mathématique de France, vol. 16, pp. 176-183, 1888.

[30] R. M. Losee, "A Gray code based ordering for documents on shelves: classification for browsing and retrieval," J. Amer. Soc. Inform. Sci., vol. 43, pp. 312-322, 1992.

[31] J. M. Ludman, "Gray codes generation for MPSK signals," IEEE Trans. on Communications, vol. COM-29, pp. 1519-1522, 1981.

[32] F. J. MacWilliams and N. J. A. Sloane, The Theory of Error-Correcting Codes. North-Holland, 1978.

[33] C. J. Mitchell, T. Etzion, and K. G. Paterson, "A method for constructing decodable de Bruijn sequences," IEEE Trans. on Inform. Theory, vol. IT-42, pp. 1472-1478, 1996.

[34] D. Richards, "Data compression and Gray-code sorting," Information Processing Letters, vol. 22, pp. 201-205, 1986.

[35] J. Robinson and M. Cohn, "Counting seqeuences," IEEE Trans. on Comput., vol. C-30, pp. 17-23, May 1981

[36] F. Ruskey, "Adjacent interchange generation of combinations," J. of Algorithms, vol. 9, pp. 162-180, 1988.

[37] C. D. Savage, "A survey of combinatorial Gray codes," SIAM Rev., vol. 39, no. 4, pp. 605-629, Dec. 1997.

[38] M. Schwartz and T. Etzion, "The structure of single-track Gray codes," IEEE Trans. on Inform. Theory, vol. 45, no. 7, pp. 2383-2396, Nov. 1999.

[39] D. Slepian, "Permutation modulation," in Proc. of the IEEE, vol. 53, no. 3, 1965, pp. 228-236.

[40] N. J. A. Sloane, "On single-deletion-correcting codes," in Codes and Designs, Ohio State University, May 2000 (Ray-Chaudhuri Festschrift), K. T. Arasu and A. Seress, Eds. Berlin: Walter de Gruyter, 2002, pp. 273-291.

[41] I. Tamo and M. Schwartz, "Correcting limited-magnitude errors in the rank-modulation scheme," IEEE Trans. on Inform. Theory, vol. 56, no. 6, pp. 2551-2560, Jun. 2010.

[42] J. Tuliani, "De Bruijn sequences with efficient decoding algorithms," Discrete Math., vol. 226, no. 1, pp. 313-336, Jan. 2001.

[43] H. Vinck, J. Haering, and T. Wadayama, "Coded M-FSK for power line communications," in Proceedings of the 2000 IEEE International Symposium on Information Theory (ISIT2000), Sorrento, Italy, 2000, p. 137.

[44] Z. Wang, A. Jiang, and J. Bruck, "On the capacity of bounded rank modulation for flash memories," in Proceedings of the 2009 IEEE International Symposium on Information Theory (ISIT2009), Seoul, Korea, Jun. 2009, pp. 1234-1238.

[45] E. Yaakobi, P. H. Siegel, and J. K. Wolf, "Buffer codes for multilevel flash memory," in Proceedings of the 2008 IEEE International Symposium on Information Theory (ISIT2008), Toronto, Canada, 2008, poster. 